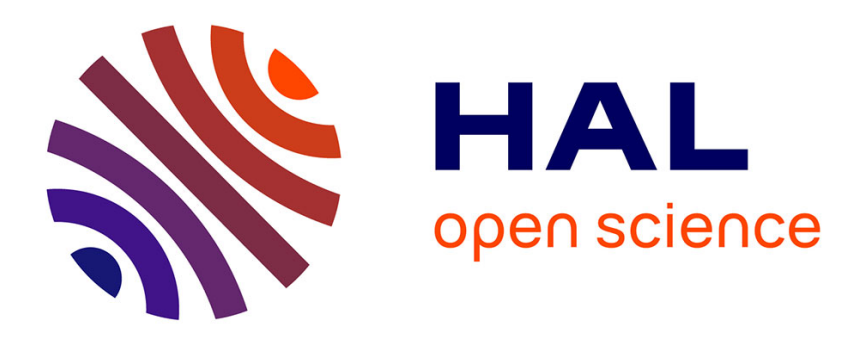

\title{
Doubloons and new q-tangent numbers
}

Dominique Foata, Guo-Niu Han

\section{To cite this version:}

Dominique Foata, Guo-Niu Han. Doubloons and new q-tangent numbers. Quarterly Journal of Mathematics, 2009, 17 p. hal-00438482

\section{HAL Id: hal-00438482 \\ https://hal.science/hal-00438482}

Submitted on 3 Dec 2009

HAL is a multi-disciplinary open access archive for the deposit and dissemination of scientific research documents, whether they are published or not. The documents may come from teaching and research institutions in France or abroad, or from public or private research centers.
L'archive ouverte pluridisciplinaire HAL, est destinée au dépôt et à la diffusion de documents scientifiques de niveau recherche, publiés ou non, émanant des établissements d'enseignement et de recherche français ou étrangers, des laboratoires publics ou privés. 
December 2, 2009

\title{
Doubloons and new $q$-tangent numbers
}

\author{
Dominique Foata and Guo-Niu Han
}

\begin{abstract}
We introduce new $q$-tangent numbers based on the Carlitz $q$-analog of the Eulerian polynomial and the so-called doubloon combinatorial set-up. Those new $q$-tangent numbers are polynomials with positive integral coefficients. They are divisible by products of binomials of the form $1+q^{i}$, the quotients being $q$-analogs of the reduced tangent numbers having an explicit combinatorial interpretation.
\end{abstract}

\section{Introduction}

In his search for an evaluation of the alternating sum $\sum_{i=1}^{m}(-1)^{i} i^{n}$ Euler [Eu1755] introduced the sequence of now called Eulerian polynomials $\left(A_{n}(t)\right)(n \geq 0)$ in the following two equivalent forms:

$$
\begin{aligned}
\frac{t-1}{t-\exp (u(t-1))}= & \sum_{n \geq 0} \frac{u^{n}}{n !} A_{n}(t) \\
=1+\frac{u}{1 !} & +\frac{u^{2}}{2 !}(t+1)+\frac{u^{3}}{3 !}\left(t^{2}+4 t+1\right) \\
& \quad+\frac{u^{4}}{4 !}\left(t^{3}+11 t^{2}+11 t+1\right)+\cdots
\end{aligned}
$$

(1.2) $\sum_{i=1}^{m} i^{n} t^{i}=\sum_{l=1}^{n}(-1)^{n+l}\left(\begin{array}{l}n \\ l\end{array}\right) \frac{t^{m+1} A_{n-l}(t)}{(t-1)^{n-l+1}} m^{l}+(-1)^{n} \frac{t\left(t^{m}-1\right)}{(t-1)^{n+1}} A_{n}(t)$.

The following infinite form of (1.2), also equivalent to both (1.1) and (1.2),

$$
\sum_{i \geq 0} t^{i}(i+1)^{n}=\frac{A_{n}(t)}{(1-t)^{n+1}} \quad(n \geq 0)
$$

is more of common usage today, while Euler's relation (1.2) seems to have been suprisingly forgotten. He also knew how to write the now called Taylor expansion of $\tan u$ as

$$
\begin{aligned}
\tan u & =\sum_{n \geq 0} \frac{u^{2 n+1}}{(2 n+1) !} T_{2 n+1} \\
& =\frac{u}{1 !}+\frac{u^{3}}{3 !} 2+\frac{u^{5}}{5 !} 16+\frac{u^{7}}{7 !} 272+\frac{u^{9}}{9 !} 7936+\frac{u^{11}}{11 !} 353792+\cdots
\end{aligned}
$$

As $\tan u=\frac{1}{i} \frac{1-e^{-2 i u}}{1+e^{-2 i u}}$, also equal to $\sum_{n \geq 1} \frac{u^{n}}{n !} i^{n-1} A_{n}(-1)$ by $(1.1)$, he derived the relations

$$
A_{2 n}(-1)=0 \quad(n \geq 1) ; \quad(-1)^{n} A_{2 n+1}(-1)=T_{2 n+1} \quad(n \geq 0) .
$$


Since Euler both Eulerian polynomials $A_{n}(t)$ and tangent numbers $T_{2 n+1}$ $(n \geq 0)$ have been given several combinatorial interpretations (see, e.g. [Ri58], [St99] and [FS70], chap. 5 for a combinatorial proof of (1.5)). Also several $q$-analogs for both polynomials have been proposed, each of them having interesting geometric properties. The purpose of this paper is to derive a $q$-analog for (1.5). This means that a sequence of polynomials $\left(A_{n}^{*}(t, q)\right)(n \geq 0)$ is to be found having the following properties:

$(P 1)$ the coefficients of each polynomial $A_{n}^{*}(t, q)$ are positive integers;

$(P 2) A_{n}^{*}(t, 1)=A_{n}(t)(n \geq 0)$

$(P 3) A_{2 n}^{*}(-1, q)=0(n \geq 1)$

$(P 4)$ the coefficients of each polynomial $(-1)^{n} A_{2 n+1}^{*}(-1, q)(n \geq 0)$ are positive integers;

(P5) $(-1)^{n} A_{2 n+1}^{*}(-1,1)=T_{2 n+1}(n \geq 0)$.

Our second goal is to provide combinatorial interpretations for those two sequences of polynomials $\left(A_{n}^{*}(t, q)\right),\left((-1)^{n} A_{2 n+1}^{*}(-1, q)\right)$, compatible with what is already known for $A_{n}(t)$ and $T_{2 n+1}$. As we now see, the polynomials $A_{n}^{*}(t, q)$, we have found, are slight variations of the classical $q$ analogs $A_{n}(t, q)$ of the Eulerian polynomials introduced by Carlitz [Ca54]. The latter polynomials may be defined by the identity

$$
\frac{A_{n}(t, q)}{(t ; q)_{n+1}}=\sum_{j \geq 0} t^{j}\left([j+1]_{q}\right)^{n} \quad(n \geq 0),
$$

where $(t ; q)_{n+1}=(1-t)(1-t q) \cdots\left(1-t q^{n}\right)$ and $[j+1]_{q}=1+q+q^{2}+\cdots+q^{n}$ are the traditional $q$-ascending factorials and $q$-analogs of the positive integers. When $q=1$, identity (1.6) is transformed into (1.3), so that

$$
A_{n}(t, 1)=A_{n}(t)
$$

Let us reproduce the first values of the polynomials $A_{n}(t, q)$ (see [Ca54], p. 336):

$$
\begin{aligned}
A_{0}(t, q)= & A_{1}(t, q)=1 ; A_{2}(t, q)=1+t q ; A_{3}(t, q)=1+2 t q(q+1)+t^{2} q^{3} \\
A_{4}(t, q)= & 1+t q\left(3 q^{2}+5 q+3\right)+t^{2} q^{3}\left(3 q^{2}+5 q+3\right)+t^{3} q^{6} ; \\
A_{5}(t, q)= & 1+t q\left(4 q^{3}+9 q^{2}+9 q+4\right)+t^{2} q^{3}\left(6 q^{4}+16 q^{3}+22 q^{2}+16 q+6\right)+ \\
& t^{3} q^{6}\left(4 q^{3}+9 q^{2}+9 q+4\right)+t^{4} q^{10} .
\end{aligned}
$$

Table 1.1. The Carlitz $q$-Eulerian Polynomials

The polynomials $A_{n}^{*}(t, q)$ which meet our expectations are defined by:

$$
\begin{aligned}
A_{2 n}^{*}(t, q) & =q^{\left(\begin{array}{c}
n \\
2
\end{array}\right)} A_{2 n}\left(t q^{-n}, q\right) ; \\
A_{2 n+1}^{*}(t, q) & =q^{\left(\begin{array}{c}
n \\
2
\end{array}\right)} A_{2 n+1}\left(t q^{-n}, q\right) ;
\end{aligned}
$$


and our new q-tangent numbers by

$$
\begin{aligned}
T_{2 n+1}(q) & =(-1)^{n} A_{2 n+1}^{*}(-1, q) \\
& =(-1)^{n} q^{\left(\begin{array}{c}
n \\
2
\end{array}\right)} A_{2 n+1}\left(-q^{-n}, q\right) .
\end{aligned}
$$

The main result of this paper is the following theorem.

Theorem 1.1. Let $T_{2 n+1}(q)$ be defined by (1.10). Then

(a) $T_{2 n+1}(q)$ is a polynomial;

(b) its coefficients are positive integers;

(c) $T_{2 n+1}(1)=T_{2 n+1}$ (the tangent number).

The proof of $(a)$ is a consequence of Proposition 1.2 further stated. The proof of $(b)$ is the most difficult part. It requires a long combinatorial development given in the next sections. The proof of $(c)$ is easy:

$$
\begin{aligned}
T_{2 n+1}(1) & =(-1)^{n} A_{2 n+1}(-1,1) \\
& =(-1)^{n} A_{2 n+1}(-1) \\
& =T_{2 n+1} .
\end{aligned}
$$

Using Table 1.1 we can determine the first values of those new $q$-tangent numbers $T_{2 n+1}(q)$ :

$$
\begin{gathered}
T_{1}(q)=1 ; T_{3}(q)=1+q ; T_{5}(q)=2+4 q+4 q^{2}+4 q^{3}+2 q^{4} ; \\
T_{7}(q)=5+17 q+29 q^{2}+39 q^{3}+46 q^{4}+46 q^{5}+39 q^{6}+29 q^{7}+17 q^{8}+5 q^{9} .
\end{gathered}
$$

Table 1.2. The new $q$-tangent numbers

Of course, we recover the traditional tangent numbers: $1,2,16,272$, by replacing $q$ by 1 . They differ from the usual $q$-tangent numbers $\tan _{2 n+1}(q)$ occurring in the expansion of the ratio of the $q$-sine by the $q$-cosine introduced by Jackson [Ja04] (also see [AG78], [Fo81], [GR90, p. 23])

$$
\sum_{n \geq 0} \frac{u^{2 n+1}}{(q ; q)_{2 n+1}} \tan _{2 n+1}(q)=\frac{\sum_{n \geq 0}(-1)^{n} u^{2 n+1} /(q ; q)_{2 n+1}}{\sum_{n \geq 0}(-1)^{n} u^{2 n} /(q ; q)_{2 n}}
$$

whose first values are $\tan _{1}(q)=1 ; \tan _{3}(q)=q+q^{2} ; \tan _{5}(q)=q^{2}+2 q^{3}+$ $3 q^{4}+4 q^{5}+3 q^{6}+2 q^{7}+q^{8} ; \tan _{7}(q)=q^{3}(1+q)^{2}\left(1+q^{2}\right)\left(1+q^{3}\right)(1+q+$ $\left.3 q^{2}+2 q^{3}+3 q^{4}+2 q^{5}+3 q^{6}+q^{7}+q^{8}\right)$.

In a subsequent paper Carlitz [Ca75] obtained the following combinatorial interpretation for the polynomial $A_{n}(t, q)$. Let $\sigma=\sigma(1) \sigma(2) \cdots \sigma(n)$ be a permutation of $12 \cdots n$. The number of descents, $\operatorname{des} \sigma$, (resp. major index, $\operatorname{maj} \sigma$,) of the permutation $\sigma$ is defined to be the number (resp. the 
sum) of all $i$ 's such that $1 \leq i \leq n-1$ and $\sigma(i)>\sigma(i+1)$. The following result

$$
A_{n}(t, q)=\sum_{\sigma \in \mathfrak{S}_{n}} t^{\operatorname{des} \sigma} q^{\operatorname{maj} \sigma} \quad(n \geq 0)
$$

is due to him. From (1.11) and the definition of $A_{2 n+1}^{*}(t, q)$ given in (1.9) it follows that

$$
A_{2 n+1}^{*}(t, q)=\sum_{\sigma \in \mathfrak{S}_{2 n+1}} t^{\operatorname{des} \sigma} q^{\mathrm{cmaj} \sigma}
$$

where cmaj $\sigma$ is the compressed major index of $\sigma$ defined by

$$
\operatorname{cmaj} \sigma=\operatorname{maj} \sigma-n \operatorname{des} \sigma+n(n-1) / 2 \text {. }
$$

Proposition 1.2. If $\sigma \in \mathfrak{S}_{2 n+1}$, then

$$
0 \leq \operatorname{cmaj} \sigma \leq n^{2}
$$

In particular, $A_{2 n+1}^{*}(t, q)$ is a polynomial of degree $(n-1)$ in $t$ and $n^{2}$ in $q$ and $T_{2 n+1}(q)$ is a polynomial (condition (a) of Theorem 1.1 holds).

Proof. We just verify:

$$
\begin{aligned}
\min _{\sigma \in \mathfrak{S}_{2 n+1}}(\operatorname{maj} \sigma-n \operatorname{des} \sigma) & =\min \left(i_{1}+i_{2}+\cdots i_{d}-n d\right) \\
& I=\left\{i_{1}, i_{2}, \ldots, i_{d}\right\} \subset[2 n] \\
& =\min \left(\left(i_{1}-n\right)+\left(i_{2}-n\right)+\cdots\left(i_{d}-n\right)\right) \\
& I=\left\{i_{1}, i_{2}, \ldots, i_{d}\right\} \subset[2 n] \\
& =(1-n)+(2-n)+\cdots(n-n)=-n(n-1) / 2 ; \\
\max _{\sigma \in \mathfrak{S}_{2 n+1}}(\operatorname{maj} \sigma-n \operatorname{des} \sigma) & =\max \left(\left(i_{1}-n\right)+\left(i_{2}-n\right)+\cdots\left(i_{d}-n\right)\right) \\
& I=\left\{i_{1}, i_{2}, \ldots, i_{d}\right\} \subset[2 n] \\
& =(n-n)+((n+1)-n)+\cdots((n+n)-n) \\
& =n(n+1) / 2 .
\end{aligned}
$$

Go back to the five properties $(P 1)-(P 5)$ we wish to fulfill: $(P 1)$ has been proved by Proposition 1.2. Also, $(P 2)$ holds since $A_{n}^{*}(t, 1)=A_{n}(t, 1)=$ $A_{n}(t)$ by (1.7). (P5) is condition $(c)$ of Theorem 1.1 and has been proved. Let us deal with $(P 3)$.

Proposition 1.3. For each $n \geq 1$ we have: $A_{2 n}^{*}(-1, q)=0$.

Proof. One possibility is to go back to the other recurrence relations for the Carlitz polynomials $A_{n}(t, q)$ and extract an analytic proof. Feasible 
but cumbersome. We can instead use the following combinatorial argument based on (1.11). Let $\sigma=\sigma(1) \sigma(2) \cdots \sigma(2 n)$ be a permutation of $12 \cdots(2 n)$ and let $\mathbf{r} \sigma$ be the reverse permutation $\mathbf{r} \sigma=\sigma(2 n) \cdots \sigma(2) \sigma(1)$. If $\sigma$ has $j$ descents in positions $d_{1}, d_{2}, \ldots, d_{j}\left(1 \leq d_{1}<d_{2}<\cdots<d_{j} \leq\right.$ $2 n-1)$, its major index, maj $\sigma$, is equal to $d_{1}+d_{2}+\cdots+d_{j}$. Hence, the major index of $\mathbf{r} \sigma$ can be evaluated as maj $\mathbf{r} \sigma=1+2+\cdots+(2 n-1)-$ $\left(n-d_{1}\right)-\left(n-d_{2}\right)-\cdots-\left(n-d_{j}\right)$. As $\operatorname{des} \mathbf{r} \sigma=2 n-1-j$, we have $-n \operatorname{des} \mathbf{r} \sigma+\operatorname{maj} \mathbf{r} \sigma=-n(2 n-1-j)+1+2+\cdots+(2 n-1)-\left(n-d_{1}\right)-$ $\left(n-d_{2}\right)-\cdots-\left(n-d_{j}\right)=-n j+d_{1}+d_{2}+\cdots+d_{j}=-n \operatorname{des} \sigma+\operatorname{maj} \sigma$. Thus $\left(-q^{-n}\right)^{\operatorname{des} \sigma} q^{\operatorname{maj} \sigma}=-\left(-q^{-n}\right)^{\operatorname{des} \mathbf{r} \sigma} q^{\operatorname{maj} \mathbf{r} \sigma}$.

There remains to prove $(P 4)$, that is, Theorem $1.1(b)$. As a matter of fact, we will prove the following stronger result.

Theorem 1.4. The ratio $d_{n}(q)=\frac{T_{2 n+1}(q)}{(1+q)\left(1+q^{2}\right) \cdots\left(1+q^{n}\right)}$ is a polynomial in $q$ with positive integral coefficients.

Our proof will be of combinatorial nature, but the problem remains open for a true analytical one, which would use the very definition of the Carlitz $q$-Eulerian polynomials $A_{n}(t, q)$, in particular (1.6), from which we can derive:

$$
\frac{A_{n}(t, q)}{(t ; q)_{n+1}}=\frac{1}{(1-q)^{n}} \sum_{k=0}^{n}\left(\begin{array}{l}
n \\
k
\end{array}\right) \frac{(-1)^{k} q^{k}}{1-t q^{k}}
$$

so that

$$
\frac{A_{2 n+1}^{*}(t, q)}{\left(t q^{-n}, q\right)_{2 n+1}}=\frac{q^{n(n-1) / 2}}{(1-q)^{2 n+1}} \sum_{k=0}^{2 n+1}\left(\begin{array}{c}
2 n+1 \\
k
\end{array}\right) \frac{(-1)^{k} q^{k}}{1-t q^{-n} q^{k}}
$$

Hence

$$
\frac{T_{2 n+1}(q)}{\left(-q^{-n}, q\right)_{2 n+1}}=\frac{(-1)^{n} q^{n(n-1) / 2}}{(1-q)^{2 n+1}} \sum_{k=0}^{2 n+1}\left(\begin{array}{c}
2 n+1 \\
k
\end{array}\right) \frac{(-1)^{k} q^{k}}{1+q^{-n} q^{k}}
$$

and

$$
\frac{T_{2 n+1}(q)}{(1+q)\left(1+q^{2}\right) \cdots\left(1+q^{n}\right)}=\frac{(-1)^{n+1}(-1 ; q)_{n+2}}{(1-q)^{2 n+1}} \sum_{k=0}^{2 n+1}\left(\begin{array}{c}
2 n+1 \\
k
\end{array}\right) \frac{(-1)^{k}}{1+q^{k-n}} .
$$

Theorem 1.4 asserts that the right-hand side of the last identity is a polynomial in $q$ with integral positive coefficients. A direct proof would be welcome.

The first values of $d_{n}(q)=T_{2 n+1}(q) /(1+q)\left(1+q^{2}\right) \cdots\left(1+q^{n}\right)$ are reproduced in Table 1.3.

$$
\begin{aligned}
& d_{0}(q)=d_{1}(q)=1 ; \quad d_{2}(q)=2+2 q ; \quad d_{3}(q)=5+12 q+12 q^{2}+5 q^{3} \\
& d_{4}(q)=14+56 q+110 q^{2}+136 q^{3}+110 q^{4}+56 q^{5}+14 q^{6} .
\end{aligned}
$$

Table 1.3. The $q$-reduced tangent polynomials 
From Theorems $1.1(c)$ and 1.4 it follows that $d_{n}(1)=T_{2 n+1} / 2^{n}$. Accordingly, the integers $d_{n}(1)$ are the reduced tangent numbers. Their exponential generating function is directly obtainable from (1.4) and reads:

$$
\begin{aligned}
\sqrt{2} \tan \frac{u}{\sqrt{2}} & =\sum_{n \geq 0} \frac{u^{2 n+1}}{(2 n+1) !} d_{n}(1) \\
& =\frac{u}{1 !} 1+\frac{u^{3}}{3 !} 1+\frac{u^{5}}{5 !} 4+\frac{u^{7}}{7 !} 34+\frac{u^{9}}{9 !} 496+\frac{u^{11}}{11 !} 353792+\cdots
\end{aligned}
$$

The combinatorial set-up used in this paper is directly inspired from the models used in our previous statistical studies ([Ha92], [Ha94], [FH00]). It can be described as follows. A doubloon of order $(2 n+1)$ is a $2 \times(n+1)$ matrix $\delta=\left(\begin{array}{c}a_{0} a_{1} \cdots a_{n} \\ b_{0} b_{1} \cdots b_{n}\end{array}\right)$ such that the word $a_{0} a_{1} \cdots a_{n} b_{n} b_{n-1} \cdots b_{0}$ is a permutation of $012 \cdots(2 n+1)$. Let $\mathcal{D}_{2 n+1}\left(\right.$ resp. $\left.\mathcal{D}_{2 n+1}^{0}\right)$ denote the set of all doubloons $\delta=\left(\begin{array}{ccc}a_{0} & a_{1} \cdots & a_{n} \\ b_{0} b_{1} \cdots b_{n}\end{array}\right)$ of order $(2 n+1)$ (resp. the subset of $\mathcal{D}_{2 n+1}$ composed of all doubloons such that $\left.a_{0}=0\right)$. When $\delta=\left(\begin{array}{cccc}0 & a_{1} & \cdots & a_{n} \\ b_{0} & b_{1} & \cdots & b_{n}\end{array}\right)$ belongs to $\mathcal{D}_{2 n+1}^{0}$, the word $\rho(\delta)=a_{1} \cdots a_{n} b_{n} b_{n-1} \cdots b_{0}$ is called the reading of $\delta$. Clearly, $\rho$ provides a bijection of $\mathcal{D}_{2 n+1}^{0}$ onto $\mathfrak{S}_{2 n+1}$. When $\delta$ belongs to $\mathcal{D}_{2 n+1}^{0}$, we simply define: $\operatorname{des} \delta=\operatorname{des} \rho(\delta), \operatorname{maj} \delta=\operatorname{maj} \rho(\delta), \operatorname{cmaj} \delta=$ $\operatorname{cmaj} \rho(\delta)$.

Now take advantage of the presentation of each doubloon as a two-row matrix $\delta=\left(\begin{array}{c}a_{0} a_{1} \cdots a_{n} \\ b_{0} b_{1} \cdots b_{n}\end{array}\right)$ for introducing the notions of interlacedness and

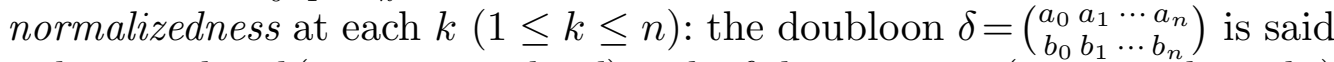
to be interlaced (resp. normalized) at $k$, if the sequence $\left(a_{k-1}, a_{k}, b_{k-1}, b_{k}\right)$ or one of its three cyclic rearrangements is monotonic increasing or decreasing (resp. decreasing). In the beginning of the next section it will be seen that out of the $4 !=24$ possible orderings of the elements $a_{k-1}, a_{k}$, $b_{k-1}, b_{k}$ there are four of them that make $\delta$ interlaced but not normalized at $k$ (relations $(I 1)-(I 4))$ and four others that make $\delta$ normalized at $k$ (relations $(I N 1)-(I N 4))$.

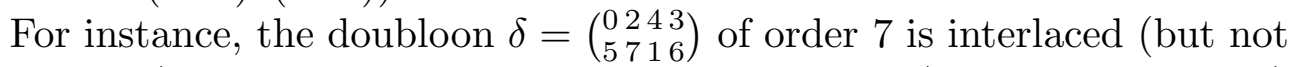
normalized) at 1, since 0257 is increasing; interlaced (but not normalized) at 2, since the cyclic rearrangement 1247 of 2471 is increasing; normalized (and also interlaced) at 3, since the cyclic rearrangement 6431 of 4316 is decreasing.

When a doubloon of order $(2 n+1)$ is interlaced (resp. normalized) at every $k=1,2, \ldots, n$, we simply say that it is interlaced (resp. normalized). Let $\mathcal{I}_{2 n+1}^{0}$ (resp. $\left.\mathcal{N}_{2 n+1}^{0}\right)$ denote the set of all doubloons from $\mathcal{D}_{2 n+1}^{0}$, which are interlaced (resp. normalized). 
Theorem 1.5. The polynomial $T_{2 n+1}(q)$ is the generating function for the set $\mathcal{I}_{2 n+1}^{0}$ of interlaced doubloons by "cmaj":

$$
T_{2 n+1}(q)=\sum_{\delta \in \mathcal{I}_{2 n+1}^{0}} q^{\mathrm{cmaj} \delta}
$$

Theorem 1.6. The following factorization holds

$$
\sum_{\delta \in \mathcal{I}_{2 n+1}^{0}} q^{\mathrm{cmaj} \delta}=(1+q)\left(1+q^{2}\right) \cdots\left(1+q^{n}\right) \sum_{\delta \in \mathcal{N}_{2 n+1}^{0}} q^{\mathrm{cmaj} \delta},
$$

so that the polynomial $d_{n}(q)$ is the generating function for the set $\mathcal{N}_{2 n+1}^{0}$ of normalized doubloons by "cmaj":

$$
d_{n}(q)=\sum_{\delta \in \mathcal{N}_{2 n+1}^{0}} q^{\mathrm{cmaj} \delta}
$$

The rest of the paper is devoted to proving the previous two theorems, which give combinatorial interpretations to $T_{2 n+1}(q)$ and $d_{n}(q)$ and evidently imply Theorems 1.1 and 1.4.

There is one normalized doubloon of order 3 , namely $\left(\begin{array}{l}03 \\ 21\end{array}\right)$, whose "cmaj" is null, the other interlaced doubloon being $\left(\begin{array}{ll}0 & 1 \\ 2 & 3\end{array}\right)$ with a "cmaj" equal to 1 . Hence, $d_{1}(q)=1$ and $T_{3}(q)=1+q$. In Table 1.4 are displayed the sixteen interlaced doubloons of order 5 with the values of their "cmaj" next to them. The top row contains the four normalized doubloons of order 5 . Hence, $d_{2}(q)=2+2 q, T_{5}(q)=(1+q)\left(1+q^{2}\right)(2+2 q)$.

$$
\begin{array}{llll}
\left(\begin{array}{lll}
0 & 5 & 3 \\
4 & 2 & 1
\end{array}\right), 0 & \left(\begin{array}{lll}
0 & 4 & 2 \\
3 & 1 & 5
\end{array}\right), 1 & \left(\begin{array}{lll}
0 & 5 & 4 \\
3 & 2 & 1
\end{array}\right), 0 & \left(\begin{array}{lll}
0 & 4 & 3 \\
2 & 1 & 5
\end{array}\right), 1 \\
\left(\begin{array}{lll}
0 & 5 & 1 \\
4 & 2 & 3
\end{array}\right), 1 & \left(\begin{array}{lll}
0 & 4 & 5 \\
3 & 1 & 2
\end{array}\right), 2 & \left(\begin{array}{lll}
0 & 5 & 1 \\
3 & 2 & 4
\end{array}\right), 1 & \left(\begin{array}{lll}
0 & 4 & 5 \\
2 & 1 & 3
\end{array}\right), 2 \\
\left(\begin{array}{lll}
0 & 2 & 3 \\
4 & 5 & 1
\end{array}\right), 3 & \left(\begin{array}{lll}
0 & 1 & 2 \\
3 & 4 & 5
\end{array}\right), 4 & \left(\begin{array}{lll}
0 & 2 & 4 \\
3 & 5 & 1
\end{array}\right), 3 & \left(\begin{array}{lll}
0 & 1 & 3 \\
2 & 4 & 5
\end{array}\right), 4 \\
\left(\begin{array}{lll}
0 & 2 & 1 \\
4 & 5 & 3
\end{array}\right), 2 & \left(\begin{array}{lll}
0 & 1 & 5 \\
3 & 4 & 2
\end{array}\right), 3 & \left(\begin{array}{lll}
0 & 2 & 1 \\
3 & 5 & 4
\end{array}\right), 2 & \left(\begin{array}{lll}
0 & 1 & 5 \\
2 & 4 & 3
\end{array}\right), 3
\end{array}
$$

Table 1.4. The set $\mathcal{I}_{5}^{0}$ of interlaced doubloons of order 5

\section{Geometry of doubloons}

Let $\delta=\left(\begin{array}{c}a_{0} a_{1} \cdots a_{n} \\ b_{0} b_{1} \cdots b_{n}\end{array}\right)$ be a doubloon and for each $k=1,2, \ldots, n$ write the four relations that make $\delta$ be normalized at $k$ (conditions (IN1)-(IN4)), and the four relations making $\delta$ be interlaced but not normalized at $k$ (conditions $(I 1)-(I 4))$ :
(IN1) $b_{k}<b_{k-1}<a_{k}<a_{k-1}$;
(I1) $a_{k}<b_{k-1}<b_{k}<a_{k-1}$;
(IN2) $b_{k-1}<a_{k}<a_{k-1}<b_{k}$;
(I2) $b_{k-1}<b_{k}<a_{k-1}<a_{k}$;
(IN3) $a_{k}<a_{k-1}<b_{k}<b_{k-1}$;
(I3) $b_{k}<a_{k-1}<a_{k}<b_{k-1}$;
(IN4) $a_{k-1}<b_{k}<b_{k-1}<a_{k}$;
(I4) $a_{k-1}<a_{k}<b_{k-1}<b_{k}$. 
We next introduce a class of transformations $\phi_{k}(0 \leq k \leq n)$ on $\mathcal{D}_{2 n+1}$, called micro flips, which simply permute the entries in a given column. For $0 \leq k \leq n$ define:

$$
\phi_{k}:\left(\begin{array}{l}
a_{0} \cdots a_{k-1} a_{k} a_{k+1} \cdots a_{n} \\
b_{0} \cdots b_{k-1} b_{k} b_{k+1} \cdots b_{n}
\end{array}\right) \mapsto\left(\begin{array}{l}
a_{0} \cdots a_{k-1} b_{k} a_{k+1} \cdots a_{n} \\
b_{0} \cdots b_{k-1} a_{k} b_{k+1} \cdots b_{n}
\end{array}\right) .
$$

Two doubloons $\delta, \delta^{\prime}$ are said to be equivalent, if there is a sequence $\phi_{i_{1}}$, $\phi_{i_{2}}, \ldots, \phi_{i_{k}}$ of micro flips such that $\delta^{\prime}=\phi_{i_{1}} \phi_{i_{2}} \cdots \phi_{i_{k}}(\delta)$.

Proposition 2.1. The following conditions hold:

(a) Each micro flip is an involution.

(b) The micro flips commute with each other.

(c) Each doubloon $\delta$ is normalized at $k$ if and only if $\phi_{k}(\delta)$ is interlaced but not normalized at $k$.

$(d)$ Each doubloon $\delta$ is normalized at $(k+1)$ if and only if $\phi_{k}(\delta)$ is interlaced but not normalized at $(k+1)$.

(e) Every doubloon equivalent to an interlaced doubloon is also interlaced.

$(f)$ Each equivalence class of interlaced doubloons contains one and only one normalized doubloon and there is an explicit algorithm for reaching it.

(g) Each equivalence class of interlaced doubloons from $\mathcal{I}_{2 n+1}^{0}$ contains $2^{n}$ doubloons.

Proof. Conditions $(a)$ and $(b)$ are obvious. For $(c)$ just observe that each doubloon $\delta$ satisfies $(I N i)$ if and only if $(I i)$ holds for $\phi_{k}(\delta)(i=$ $1,2,3,4)$. For (d) verify that condition (IN1) (resp. (IN2), resp. (IN3), resp. (IN4)), with $(k+1)$ replacing $k$, holds if and only if (I3) (resp. $(I 4)$, resp. (I1), resp. $(I 2))$, with $(k+1)$ replacing $k$, holds for $\phi_{k}(\delta)$. Condition $(e)$ is a consequence of $(c)$ and $(d)$. For $(f)$ let $l$ be the smallest

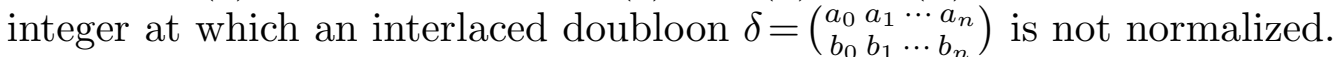
By $(c)$ the doubloon $\phi_{l}(\delta)$ is normalized at $1,2, \ldots, l$. Continue the process until reaching a doubloon normalized at all integers $1,2, \ldots, n$. Finally, $(g)$ follows from the fact that each equivalence class can be generated from an interlaced doubloon $\delta$ from the set $\mathcal{I}_{2 n+1}^{0}$ by applying the $n$ involutions $\phi_{1}, \phi_{2}, \ldots, \phi_{n}$ to $\delta$, independently.

For comparing two adjacent biletters $\left(\begin{array}{l}a_{k-1} \\ b_{k-1}\end{array}\right)$ and $\left(\begin{array}{l}a_{k} \\ b_{k}\end{array}\right)(1 \leq k \leq n)$ of a doubloon $\delta=\left(\begin{array}{cccc}a_{0} & a_{1} & \cdots & a_{n} \\ b_{0} & b_{1} & \cdots & b_{n}\end{array}\right)$ we define: $\operatorname{shape}_{k}(\delta)=\left(\begin{array}{l}> \\ >\end{array}\right)\left(\operatorname{resp} \cdot\left(\begin{array}{l}> \\ <\end{array}\right)\right.$, resp. $\left(\begin{array}{l}< \\ <\end{array}\right)$, resp. $\left.\left(\begin{array}{l}< \\ >\end{array}\right)\right)$, if $a_{k-1}>a_{k}$ and $b_{k-1}>b_{k}$ (resp. $a_{k-1}>a_{k}$ and $b_{k-1}<b_{k}$, resp. $a_{k-1}<a_{k}$ and $b_{k-1}<b_{k}$, resp. $a_{k-1}<a_{k}$ and $\left.b_{k-1}>b_{k}\right)$. We also define shape $_{n+1}(\delta)=\vee$ or $\wedge$ depending on whether $a_{n}>b_{n}$ or $a_{n}<b_{n}$. The word $\operatorname{shape}_{1}(\delta) \operatorname{shape}_{2}(\delta) \cdots$ shape $_{n}(\delta)$ is called the shape of $\delta$ and denoted by shape $\delta$.

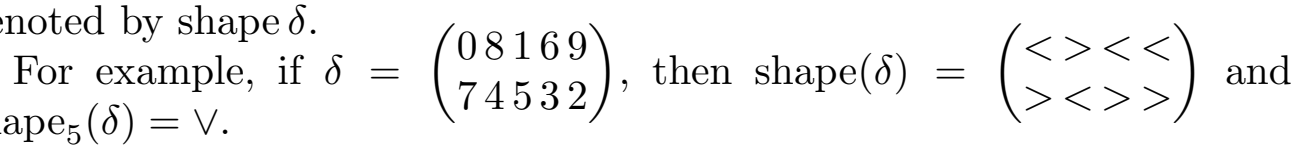


Now go back to the conditions (INi)'s and ( $I i)$ 's and determine shape ${ }_{k}$ in each one of those eight cases. We obtain:

$$
\begin{aligned}
& (I N 1)\left(\begin{array}{l}
> \\
>
\end{array}\right) ; \quad(I N 2)\left(\begin{array}{l}
> \\
<
\end{array}\right) ; \quad(I N 3)\left(\begin{array}{l}
> \\
>
\end{array}\right) ; \quad(I N 4)\left(\begin{array}{l}
< \\
>
\end{array}\right) ; \\
& (I 1)\left(\begin{array}{l}
> \\
<
\end{array}\right) ; \quad(I 2)\left(\begin{array}{l}
< \\
<
\end{array}\right) ; \quad(I 3)\left(\begin{array}{l}
< \\
>
\end{array}\right) ; \quad(I 4)\left(\begin{array}{l}
< \\
<
\end{array}\right) \text {. }
\end{aligned}
$$

As $(I i)$ holds for $\delta$ whenever $(I N i)$ does for $\phi_{k}(\delta)$, we can express the change of shape $_{k}$ when $\phi_{k}$ is applied to $\delta$ by the diagram displayed in Fig. 2.1.

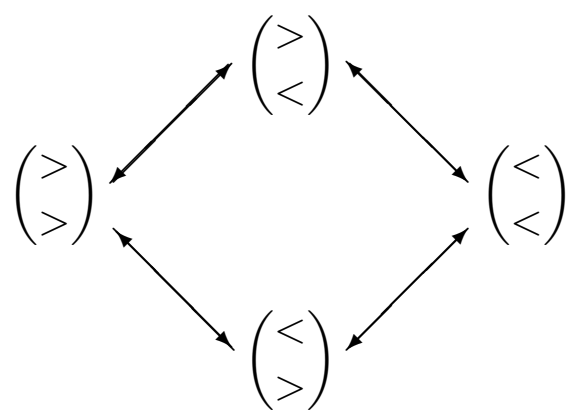

Fig. 2.1. The interlaced case

The same study is to be done for each non interlaced doubloon. There are sixteen reorderings of the sequence $\left(a_{k-1}, a_{k}, b_{k-1}, b_{k}\right)$ that make the doubloon $\delta$ non-interlaced at $k$. In Fig. 2.2 only eight of them have been listed. As non-interlacedness is preserved under $\phi_{k}$ by Proposition $2.1(c)$, the other eight are indeed obtained by applying the micro flip $\phi_{k}$. Again, the content ot the table may be summarized by the diagram displayed in Fig. 2.3. Notice that each shape may be preserved under $\phi_{k}$ (symbolized by the fixed point $\bigcirc$ ) and the only possible change occurs between the

\begin{tabular}{|c|c|c|c|}
\hline case & : & $\operatorname{shape}_{k}(\delta)$ & $\operatorname{shape}_{k} \phi_{k}(\delta)$ \\
\hline$b_{k}<b_{k-1}<a_{k-1}<a_{k}$ & & & \\
\hline$a_{k-1}<a_{k}<b_{k}<b_{k-1}$ & : & & \\
\hline$b_{k-1}<b_{k}<a_{k}<a_{k-1}$ & : & & \\
\hline$a_{k}<a_{k-1}<b_{k-1}<b_{k}$ & : & 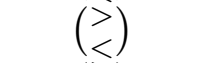 & \\
\hline$b_{k}<a_{k}<b_{k-1}<a_{k-1}$ & : & & \\
\hline$a_{k}<b_{k}<a_{k-1}<b_{k-1}$ & : & & \\
\hline$b_{k-1}<a_{k-1}<b_{k}<a_{k}$ & : & $\left(\begin{array}{l}< \\
<\end{array}\right)$ & $\left(\begin{array}{l}< \\
<\end{array}\right)$ \\
\hline$a_{k-1}<b_{k-1}<a_{k}<b_{k}$ & : & $\left(\begin{array}{l}< \\
<\end{array}\right)$ & $\left(\begin{array}{l}< \\
<\end{array}\right)$ \\
\hline
\end{tabular}
shapes $\left(\begin{array}{l}> \\ <\end{array}\right)$ and $\left(\begin{array}{l}< \\ >\end{array}\right)$.

Fig. 2.2. The sixteen non interlaced orderings 
Finally, we introduce the macro flip $\Phi_{k}$ as

$$
\Phi_{k}(\delta)=\phi_{k} \phi_{k+1} \cdots \phi_{n}(\delta) \text {. }
$$

Again, the macro flip $\Phi_{k}$ is an involution and also preserves interlacedness.

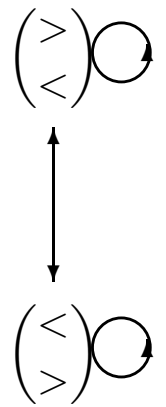

Fig. 2.3. The non-interlaced case

\section{Statistics on shapes}

The next step is to study the action of the involution $\Phi_{k}$ onto the pair of statistics (des, cmaj). As those two statistics essentially depend on the doubloon shapes, it is convenient to study the action on the shapes themselves. To that end we introduce the notion of full shape in the following manner. If $\delta$ belongs to $\mathcal{I}_{2 n+1}^{0}$, its full shape is obtained by juxtaposing shape $_{n+1}(\delta)$ to the right of its shape, as defined just after the proof of Proposition 2.1. For example, the full shape of the interlaced doubloon

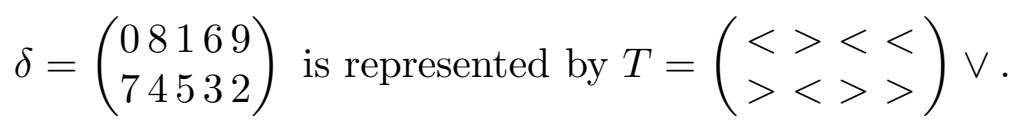

The full shape $T$ of a doubloon $\delta$ from $\mathcal{I}_{2 n+1}^{0}$ has then $n$ columns with two signs, followed by one single symbol. The statistics "des", "maj" and "cmaj" for the full shape $T$ are defined by

$\operatorname{des} T=\operatorname{des} \rho(\delta), \quad \operatorname{maj} T=\operatorname{maj} \rho(\delta) \quad$ and $\quad \operatorname{cmaj} T=\operatorname{cmaj} \rho(\delta)$.

If $\delta=\left(\begin{array}{cccc}0 & a_{1} & \ldots & a_{n} \\ b_{0} & b_{1} & \ldots & b_{n}\end{array}\right)$ and if the $k$-th column of the full shape $T$ of $\delta$ is $\left(\begin{array}{l}> \\ <\end{array}\right)(1 \leq$ $k \leq n)$, this means that $a_{k-1}>a_{k}$ and $b_{k-1}<b_{k}$. Furthermore, $b_{k}$ is the $(2 n-k+1)$-st term of the permutation $\rho(\delta)=a_{1} \cdots a_{k-1} a_{k} \cdots b_{k} b_{k-1} \cdots b_{0}$, so that the contribution of that $k$-th column to maj $T$ is $k-1+(2 n-k+1)=$ $2 n$. The remark will be used in the next lemmas.

Lemma 3.1. Let $T$ be a full shape and $S$ be obtained from $T$ by permuting two symbols in the same column. Then

$$
(-1)^{\operatorname{des} T}=(-1)^{\operatorname{des} S} \text { and } \quad \operatorname{cmaj} T=\operatorname{cmaj} S .
$$

Proof. Only one case is to consider. Suppose that the $k$-th column of $T$ (resp. of $S$ ) is $\left(\begin{array}{l}> \\ <\end{array}\right)$ (resp. $\left(\begin{array}{l}< \\ >\end{array}\right)$ ). Clearly, $\operatorname{des} T=\operatorname{des} S+2$. Moreover, $\operatorname{maj} T=\operatorname{maj} S+2 n$. Hence, $\operatorname{cmaj} T=\operatorname{maj} T-n \operatorname{des} T+n(n-1) / 2=$ maj $S+2 n-n(\operatorname{des} S+2)+n(n-1) / 2=\operatorname{cmaj} S$. 
Lemma 3.2. Let $T$ be a full shape and $S$ be obtained from $T$ by changing the value of the rightmost single symbol. Then

$$
(-1)^{\operatorname{des} T}=-(-1)^{\operatorname{des} S} \quad \text { and } \quad \operatorname{cmaj} T=\operatorname{cmaj} S .
$$

Proof. Suppose that the rightmost single symbol of $T$ (resp. of $S$ ) is $\vee$ (resp. $\wedge$ ). Clearly, $\operatorname{des} T=\operatorname{des} S+1$. Since

$$
\begin{aligned}
& \operatorname{cmaj} T=(\cdots+n+\cdots)-n \operatorname{des} T+n(n-1) / 2, \\
& \operatorname{cmaj} S=(\cdots+0+\cdots)-n \operatorname{des} S+n(n-1) / 2,
\end{aligned}
$$

we have $\operatorname{cmaj} T=\operatorname{cmaj} S$.

Lemma 3.3. Let $T$ be a full shape, whose $k$-th column is $\left(\begin{array}{l}> \\ <\end{array}\right)$ or $\left(\begin{array}{l}< \\ >\end{array}\right)$. Let $S$ be obtained from $T$ by changing the $k$-th column into $\left(\begin{array}{l}> \\ >\end{array}\right)$. Then

$$
(-1)^{\operatorname{des} T}=-(-1)^{\operatorname{des} S} \quad \text { and } \quad \operatorname{cmaj} T-\operatorname{cmaj} S=n-i+1 .
$$

Proof. If the $k$-th column of $T$ is $\left(\begin{array}{l}> \\ <\end{array}\right)$, then $\operatorname{des} T=\operatorname{des} S+1$. Moreover, with $j=2 n-k+1$ we have:

$$
\begin{aligned}
\operatorname{cmaj} T & =(\cdots+k-1+\cdots+j+\cdots)-n \operatorname{des} T+n(n-1) / 2 \\
& =(\cdots+k-1+\cdots+0+\cdots)+j-n(\operatorname{des} S+1)+n(n-1) / 2 \\
& =\operatorname{cmaj} S+j-n=\operatorname{cmaj} S+n-k+1 .
\end{aligned}
$$

For the second case we just appeal to Lemma 3.1.

Lemma 3.4. Let $T$ be a full shape, whose $k$-th column is $\left(\begin{array}{l}< \\ <\end{array}\right)$. Let $S$ be obtained from $T$ by changing the $k$-th column into $\left(\begin{array}{l}> \\ <\end{array}\right)$ or $\left(\begin{array}{l}< \\ >\end{array}\right)$. Then

$$
(-1)^{\operatorname{des} T}=-(-1)^{\operatorname{des} S} \quad \text { and } \quad \operatorname{cmaj} T-\operatorname{cmaj} S=n-k+1 .
$$

Proof. If the $k$-th column is changed into $\left(\begin{array}{l}> \\ <\end{array}\right)$, then $\operatorname{des} T=\operatorname{des} S-1$. With $j=2 n-k+1$ we have

$$
\begin{aligned}
\operatorname{cmaj} T & =(\cdots+0+\cdots+j+\cdots)-n \operatorname{des} T+n(n-1) / 2 \\
& =(\cdots+k-1+\cdots+j+\cdots)-k+1-n(\operatorname{des} S-1)+n(n-1) / 2 \\
& =\operatorname{cmaj} S+n-k+1
\end{aligned}
$$

Again it is sufficient to appeal to Lemma 3.1 to cover the second case.

The macro flips $\Phi_{k}$ have been defined in (2.1). They are involutions and preserve the interlacedness. They play a crucial role in the next theorem. 


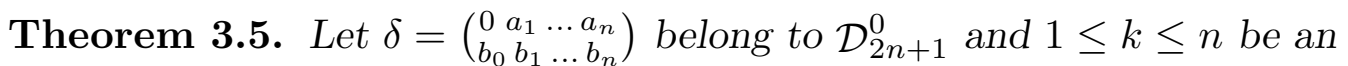
integer.

(1) If $\delta$ is normalized at $k$, then

$$
\left\{\begin{array}{l}
(-1)^{\operatorname{des} \Phi_{k}(\delta)}=(-1)^{\operatorname{des} \delta} \\
\operatorname{cmaj} \Phi_{k}(\delta)-\operatorname{cmaj} \delta=n-k+1
\end{array}\right.
$$

(2) If $\delta$ is non-interlaced at $k$, then

$$
\left\{\begin{array}{l}
(-1)^{\operatorname{des} \Phi_{k}(\delta)}=-(-1)^{\operatorname{des} \delta} \\
\operatorname{cmaj} \Phi_{k}(\delta)=\operatorname{cmaj} \delta
\end{array}\right.
$$

Proof. Apply the macro flip $\Phi_{k}=\phi_{k} \cdots \phi_{n}$ to $\delta$ and compare the shapes of $\delta$ and $\Phi_{k}(\delta)$. First, $\phi_{n}$ leaves "cmaj" invariant and changes the parity of "des" by Lemma 3.2. Next, $\phi_{n-1}, \ldots, \phi_{i+1}$ leave both "des" and "cmaj" invariant by Lemma 3.1, so that $(-1)^{\operatorname{des} \Phi_{k+1}(\delta)}=-(-1)^{\operatorname{des} \delta}$ and $\operatorname{cmaj} \Phi_{k+1}(\delta)=\operatorname{cmaj} \delta$. Note that $\operatorname{shape}_{k} \delta=\operatorname{shape}_{k} \phi_{k+1} \cdots \phi_{n}(\delta)$, as $\phi_{n}$, $\ldots, \phi_{k+1}$ have no action on the columns of rank $(k-1)$ and $k$ (the $k$-th and $(k+1)$-st columns). Also shape $\phi_{k}(\delta)=\operatorname{shape}_{k} \phi_{k+1} \cdots \phi_{n} \phi_{k}(\delta)=$ $\operatorname{shape}_{k} \phi_{k} \phi_{k+1} \cdots \phi_{n}(\delta)=\operatorname{shape}_{k} \Phi_{k}(\delta)$.

In case (1) there are two possibilities: either $\operatorname{shape}_{k} \delta=\left(\begin{array}{l}> \\ >\end{array}\right)$ (cases $(I N 1)$ and $(I N 3))$, or shape $\phi_{k}(\delta)=\left(\begin{array}{l}< \\ <\end{array}\right)(\operatorname{cases}(I N 2)$ and $(I N 4))$. When $\operatorname{shape}_{k} \delta=\left(\begin{array}{l}> \\ >\end{array}\right)\left(\right.$ resp. shape $\left._{k} \phi_{k}(\delta)=\left(\begin{array}{l}< \\ <\end{array}\right)\right)$, then

$$
\operatorname{shape}_{k} \Phi_{k}(\delta)=\operatorname{shape}_{k} \phi_{k}(\delta)=\left(\begin{array}{l}
< \\
>
\end{array}\right) \text { or }\left(\begin{array}{l}
> \\
<
\end{array}\right)
$$

according to Fig. 2.1 (resp. shape $_{k} \Phi_{k}(\delta)=\operatorname{shape}_{k} \phi_{k}(\delta)=\left(\begin{array}{l}< \\ <\end{array}\right)$ ). By Lemma 3.3 (resp. Lemma 3.4) the parity of "des" is modified when going from $\Phi_{k}(\delta)$ to $\phi_{k} \Phi_{k}(\delta)=\Phi_{k+1}(\delta)$ and $\operatorname{cmaj} \Phi_{k}(\delta)-\operatorname{cmaj} \Phi_{k+1}(\delta)=n-$ $k+1$. Hence $(-1)^{\operatorname{des} \Phi_{k}(\delta)}=-(-1)^{\operatorname{des} \Phi_{k+1}(\delta)}=(-1)^{\operatorname{des} \delta}$ and cmaj $\Phi_{k}(\delta)=$ $\operatorname{cmaj} \Phi_{k+1}(\delta)+n-k+1=\operatorname{cmaj} \delta+n-k+1$.

In case 2 look at Fig. 2.3 and apply Lemmas 3.1 and 3.2. The only change is the parity of "des."

\section{Proofs of Theorems $\mathbf{1 . 5}$ and $\mathbf{1 . 6}$}

Let $\delta$ belong to $\mathcal{D}_{2 n+1}^{0}$ and $\sigma=\rho(\delta)$ be its reading. Recall that the statistics "des", "maj" and "cmaj" for $\delta$ are defined by

$$
\operatorname{des} \delta=\operatorname{des} \sigma, \quad \operatorname{maj} \delta=\operatorname{maj} \sigma \quad \text { and } \quad \operatorname{cmaj} \delta=\operatorname{cmaj} \sigma .
$$

From the definition of $A_{2 n+1}^{*}(t, q)$ (given in (1.12)) and the fact that $\rho$ is a bijection of $\mathcal{D}_{2 n+1}^{0}$ onto $\mathfrak{S}_{2 n+1}$ we can write: 


$$
\begin{aligned}
A_{2 n+1}^{*}(t, q) & =\sum_{\delta \in \mathcal{D}_{2 n+1}^{0}} t^{\mathrm{des} \delta} q^{\mathrm{cmaj} \delta} \\
& =\sum_{\delta \in \mathcal{I}_{2 n+1}^{0}} t^{\mathrm{des} \delta} q^{\mathrm{cmaj} \delta}+\sum_{\delta \in \mathcal{D}_{2 n+1}^{0} \backslash \mathcal{I}_{2 n+1}^{0}} t^{\mathrm{des} \delta} q^{\mathrm{cmaj} \delta}
\end{aligned}
$$

so that

$$
\begin{aligned}
T_{2 n+1}(q) & =(-1)^{n} A_{2 n+1}^{*}(-1, q) \\
& =\sum_{\delta \in \mathcal{I}_{2 n+1}^{0}}(-1)^{n+\operatorname{des} \delta} q^{\mathrm{cmaj} \delta}+\sum_{\delta \in \mathcal{D}_{2 n+1}^{0} \backslash \mathcal{I}_{2 n+1}^{0}}(-1)^{n+\operatorname{des} \delta} q^{\mathrm{cmaj} \delta} .
\end{aligned}
$$

Lemma 4.1. Let $\delta \in \mathcal{I}_{2 n+1}^{0}$. Then

$$
(-1)^{\operatorname{des} \delta}=(-1)^{n}
$$

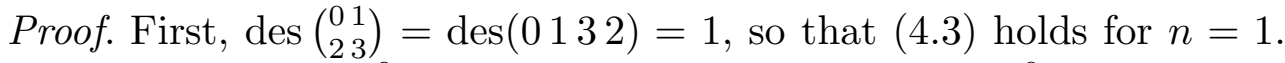
Let $n \geq 2$ and $\delta=\left(\begin{array}{c}0 \ldots a_{n-1} a_{n} \\ b_{0} \ldots b_{n-1} b_{n}\end{array}\right) \in \mathcal{I}_{2 n+1}^{0}$. Then $\delta^{\prime}=\left(\begin{array}{c}0 \ldots a_{n-1} \\ b_{0} \ldots b_{n-1}\end{array}\right)$ is an interlaced doubloon on the alphabet $[0,2 n+1] \backslash\left\{a_{n}, b_{n}\right\}$ (instead of $[0,2 n-$ 1]) and $\operatorname{des} \delta^{\prime}=(-1)^{n-1}$ by induction. As $\delta$ is interlaced at $n$, the eight orderings to consider for the sequence $\left(a_{n-1}, a_{n}, b_{n-1}, b_{n}\right)$ are the eight orderings $(I N i),(I i)(i=1,2,3,4)$ described in the beginning of Section 2 . If $a_{n-1}<b_{n-1}$, then $\operatorname{des} \delta=\operatorname{des} \delta^{\prime}+\operatorname{des}\left(a_{n-1} a_{n} b_{n} b_{n-1}\right)=\operatorname{des} \delta^{\prime}+1$. If $a_{n-1}>b_{n-1}$, then $\operatorname{des} \delta=\delta^{\prime}-1+\operatorname{des}\left(a_{n-1} a_{n} b_{n} b_{n-1}\right)=\operatorname{des} \delta^{\prime}-1+2=$ $\operatorname{des} \delta^{\prime}+1$.

From (4.2) and (4.3) we deduce:

$$
T_{2 n+1}(q)=\sum_{\delta \in \mathcal{I}_{2 n+1}^{0}} q^{\mathrm{cmaj} \delta}+\sum_{\delta \in \mathcal{D}_{2 n+1}^{0} \backslash}(-1)^{n+\operatorname{des} \delta} q^{\mathrm{cmaj} \delta} .
$$

Theorem 1.5 will be proved once we construct a sign-reversing involution on $\mathcal{D}_{2 n+1}^{0} \backslash \mathcal{I}_{2 n+1}^{0}$ that makes the second sum vanish.

The sign-reversing involution. For each doubloon $\delta=\left(\begin{array}{c}a_{0} a_{1} \ldots a_{n} \\ b_{0} b_{1} \ldots b_{n}\end{array}\right)$ from the set $\mathcal{D}_{2 n+1}^{0} \backslash \mathcal{I}_{2 n+1}^{0}$ there exists at least one integer $k$ at which $\delta$ is noninterlaced. Let $k(\delta)$ be the smallest integer satisfying that condition.

Theorem 4.2. The mapping $\Phi_{k(\delta)}: \delta \mapsto \Phi_{k(\delta)}(\delta)$ is a sign-reversing involution on $\mathcal{D}_{2 n+1}^{0} \backslash \mathcal{I}_{2 n+1}^{0}$.

Proof. This is an immediate consequence of Theorem 3.5, formulas (3.2). 
Proof of Theorem 1.6. Let $\delta$ be a normalized doubloon from $\mathcal{N}_{2 n+1}^{0}$. By Proposition 2.1 the set of all the $2^{n}$ interlaced doubloons equivalent to $\delta$ may be expressed as

$$
\left\{\Phi_{i_{1}} \Phi_{i_{2}} \cdots \Phi_{i_{k}}(\delta): 1 \leq i_{1}<i_{2}<\cdots<i_{k} \leq n\right\} .
$$

As $\Phi_{i_{k}}(\delta)\left(\operatorname{resp} . \Phi_{i_{k-1}} \Phi_{i_{k}}(\delta), \ldots\right.$, resp. $\left.\Phi_{i_{2}} \cdots \Phi_{i_{k-1}} \Phi_{i_{k}}(\delta)\right)$ is normalized at $i_{k-1}$, (resp. at $i_{k-2}, \ldots$, resp. at $i_{1}$ ), it follows from (3.2) that

$$
\begin{aligned}
\operatorname{cmaj} \Phi_{i_{1}} \Phi_{i_{2}} \cdots \Phi_{i_{k}}(\delta) & =\operatorname{cmaj} \Phi_{i_{2}} \cdots \Phi_{i_{k}}(\delta)+n-i_{1}+1 \\
& =\operatorname{cmaj} \Phi_{i_{3}} \cdots \Phi_{i_{k}}(\delta)+n-i_{2}+1+n-i_{1}+1 \\
& =\cdots \\
& =\operatorname{cmaj} \delta+n-i_{k}+1+\cdots+n-i_{1}+1 .
\end{aligned}
$$

Hence

$$
\begin{aligned}
\sum_{\alpha \text { equiv } \delta} q^{\text {cmaj } \alpha} & =\sum q^{\text {cmaj } \Phi_{i_{1}} \Phi_{i_{2}} \cdots \Phi_{i_{k}}(\delta)} \quad\left(1 \leq i_{1}<i_{2}<\cdots<i_{k} \leq n\right) \\
& =q^{\operatorname{cmaj} \delta} \sum q^{n-i_{k}+1+\cdots+n-i_{1}+1} \quad\left(1 \leq i_{1}<\cdots<i_{k} \leq n\right) \\
& =q^{\operatorname{cmaj} \delta}(1+q)\left(1+q^{2}\right) \cdots\left(1+q^{n}\right)
\end{aligned}
$$

By (4.4) we can write

$$
\begin{aligned}
T_{2 n+1}(q) & =(-1)^{n} A_{2 n+1}^{*}(-1, q) \\
& =\sum_{\alpha \in \mathcal{I}_{2 n+1}^{0}} q^{\mathrm{cmaj} \alpha} \\
& =\sum_{\delta \in \mathcal{N}_{2 n+1}^{0}} \sum_{\alpha \text { equiv } \delta} q^{\mathrm{cmaj} \alpha} \\
& =(1+q)\left(1+q^{2}\right) \cdots\left(1+q^{n}\right) \sum_{\delta \in \mathcal{N}_{2 n+1}^{0}} q^{\mathrm{cmaj} \delta} .
\end{aligned}
$$

\section{Constant terms and Catalan numbers}

Referring to Table 1.2 we see that $T_{1}(0)=T_{3}(0)=1, T_{5}(0)=2$, $T_{7}(0)=5$ and we would find $T_{9}(0)=14$. The sequence $1,1,2,5,14$ is indeed the beginning of the sequence of Catalan numbers. This is true for all $n$ as stated in the next theorem.

Theorem 5.1. For all $n \geq 0$

$$
\left.T_{2 n+1}(0)=\frac{1}{n+1}\left(\begin{array}{c}
2 n \\
n
\end{array}\right) \text { (the Catalan number }\right) .
$$


Proof. From (1.16) $T_{2 n+1}(0)=\#\left\{\sigma \in \mathfrak{S}_{2 n+1}, \operatorname{cmaj} \sigma=0\right\}$. Let $\left\{i_{1}, i_{2}, \ldots, i_{d}\right\}$ be the descent set of a permutation $\sigma$ from $\mathfrak{S}_{2 n+1}$. Then,

$$
\begin{aligned}
\operatorname{maj} \sigma-n \operatorname{des} \sigma & =\left(i_{1}+i_{2}+\cdots i_{d}-n d\right) \\
& =\left(i_{1}-n\right)+\left(i_{2}-n\right)+\cdots\left(i_{d}-n\right) \\
& =-n(n-1) / 2 \\
& =(1-n)+(2-n)+\cdots((n-1)-n)+(n-n) \\
& =(1-n)+(2-n)+\cdots((n-1)-n) .
\end{aligned}
$$

Accordingly, the descent set of $\sigma$ is either $[n]=\{1,2, \ldots, n\}$ by $(5.2)$, or $[n-1]=\{1,2, \ldots, n-1\}$ by (5.3). The number of permutations of order $2 n+1$ whose descent set is $[n]$ (resp. $[n-1])$ is equal to $\left(\begin{array}{c}2 n \\ n\end{array}\right)\left(\operatorname{resp} .\left(\begin{array}{c}2 n \\ n-1\end{array}\right)\right)$. Hence,

$$
\begin{aligned}
T_{2 n+1}(0) & =(-1)^{n} \sum_{\sigma \in \mathfrak{S}_{2 n+1}}(-1)^{\operatorname{des} \sigma} 0^{\mathrm{cmaj} \sigma} \\
& =\left(\begin{array}{c}
2 n \\
n
\end{array}\right)-\left(\begin{array}{c}
2 n \\
n-1
\end{array}\right)=\frac{1}{n+1}\left(\begin{array}{c}
2 n \\
n
\end{array}\right) \cdot \square
\end{aligned}
$$

As a summary we have:

$$
\begin{aligned}
T_{2 n+1}(1) & =T_{2 n+1}(\text { the tangent number }) \\
T_{2 n+1}(0)=d_{n}(0) & \left.=\frac{1}{n+1}\left(\begin{array}{c}
2 n \\
n
\end{array}\right) \text { (the Catalan number }\right) \\
d_{n}(1) & =t_{n}(\text { the reduced tangent number }) .
\end{aligned}
$$

Remark. Each doubloon $\delta=\left(\begin{array}{ccc}0 & a_{1} \cdots a_{n} \\ b_{0} b_{1} \cdots b_{n}\end{array}\right)$ such that cmaj $\delta=0$ has a full shape of the form $\left(\begin{array}{c}\langle>>\cdots> \\ >>>\cdots>\end{array}\right) \vee$ or $\left(\begin{array}{c}<>>\cdots> \\ >>>\cdots>\end{array}\right) \wedge$. As $\delta$ is interlaced at every $k=1,2, \ldots, n$, the following inequalities $a_{1}>b_{0}>b_{1}, a_{1}>a_{2}>b_{1}>b_{2}$, $\ldots, a_{n-1}>a_{n}>b_{n-1}>b_{n}$ hold and may be represented by the poset:

$$
\begin{aligned}
& n=a_{1}>a_{2}>\cdots>a_{n} \\
& \begin{array}{lllll} 
& \vee & \cdots & \cdots
\end{array} \\
& b_{0}>b_{1}>\cdots>b_{n-1}=1
\end{aligned}
$$

This establishes a bijection between those doubloons and the above posets, which belong to the list of combinatorial models counted by the Catalan numbers [St99, p. 227]. 


\section{Concluding remarks}

Besides the traditional $q$-tangent number, $\tan _{2 n+1}(q)$, already mentioned in the Introduction, other $q$-analogs of the tangent have been proposed, in particular by Prodinger [Pr00, Pr08] (also see [Fu00]) and Han et al. [HRZ01]. For those two cases the emphasis has been the search for adequate continued fraction expansions of those new functions.

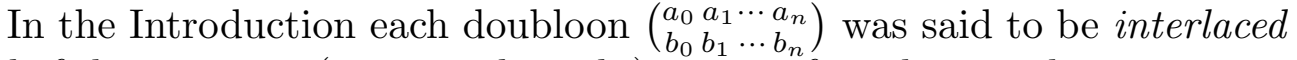
at $k$ if the sequence $\left(a_{k-1}, a_{k}, b_{k-1}, b_{k}\right)$ or one of its three cyclic rearrangements was monotonic increasing or decreasing. Another equivalent definition that was successfully used in [Ha94] is based on the notion of cyclic interval. The cyclic interval $\llbracket a_{k-1}, b_{k-1} \llbracket$ is defined as the semi-open interval $\left[a_{k-1}, b_{k-1}\right)$ if $a_{k-1}<b_{k-1}$ and as the union $\left[a_{k-1},+\infty\right) \cup\left(-\infty, b_{k-1}\right)$ otherwise. It is readily seen that the doubloon is interlaced at $k$ if and only if exactly one of the entries $a_{k}, b_{k}$ belongs to the cyclic interval $\llbracket a_{k-1}, b_{k-1} \llbracket$.

The doubloon model described in this paper serves to interpret the two $q$-analogs $T_{2 n+1}(q)$ of $T_{2 n+1}$ and $d_{n}(q)$ of $t_{n}$. A refinement of the doubloon model will be proposed in our next paper [FH08]; it will provide a refinement $d_{n, j}(q)$ of the polynomials $d_{n}(q)$, so that identities (5.5) and (5.6) will be extended to classical double-digit sequences of numbers: Catalan triangle [S107, sequence A009766] and Poupard triangle [Po89].

\section{References}

[AG78] Andrews, George E.; Gessel, Ira, Divisibility properties of the $q$-tangent numbers, Proc. Amer. Math. Soc., 68 (1978), pp. 380-384.

[Ca54] Carlitz, Leonard, $q$-Bernoulli and Eulerian numbers, Trans. Amer. Math. Soc., 76 (1954), pp. 332-350.

[Ca75] Carlitz, Leonard, A combinatorial property of $q$-Eulerian numbers, Amer. Math. Monthly, 82 (1975), pp. 51-54.

[Eul1755] Euler, Leonhard, Institutiones calculi differentialis cum eius usu in analysi finitorum ac Doctrina serierum, Academiae Imperialis Scientiarum Petropolitanae, St. Petersbourg, 1755, chap. VII ("Methodus summandi superior ulterius promota").

[FH00] Foata, Dominique; Han, Guo-Niu, Word straightening and $q$-Eulerian Calculus, Contemporary Mathematics, $q$-Series from a Contemporary Perspective, AMS, M. E. H. Ismail, D. W. Stanton Ed., 254, 2000, 141-156.

[FH08] Foata, Dominique; Han, Guo-Niu, The doubloon polynomial triangle, to appear in Ramanujan J., 2008, 19 p.

[Fo81] Foata, Dominique, Further divisibility properties of the $q$-tangent numbers, Proc. Amer. Math. Soc., 81 (1981), pp. 143-148.

[FS70] Foata, Dominique; Schützenberger, Marcel-Paul, Théorie géométrique des polynômes eulériens, Lecture Notes in Mathematics, 138, Berlin, Springer-Verlag, 1970. (http://igd.univ-lyon1.fr/〜slc/books/index.html). 
[Fu00] Fulmek, Markus, A continued fraction expansion for a $q$-tangent function, Sém. Lothar. Combin., B45b (2000), 3pp.

[GR90] Gasper, George; Rahman, Mizan, Basic hypergeometric series, Encyclopedia of Math. and its Appl. 35, Cambridge Univ. Press, Cambridge, 1990.

[Ha92] Han, Guo-Niu, Calcul Denertien, Thèse de Doctorat, Publ. l'I.R.M.A., Strasbourg, 476/TS-29, 1991, 119 pages. http://www-irma.u-strasbg.fr/ guoniu.

[Ha94] Han, Guo-Niu, Une transformation fondamentale sur les réarrangements de mots, Adv. in Math., 105(1) (1994), pp. 26-41.

[HRZ01] Han, Guo-Niu; Randrianarivony, Arthur; Zeng, Jiang, Un autre $q$-analogue des nombres d'Euler, The Andrews Festschrift. Seventeen Papers on Classical Number Theory and Combinatorics, D. Foata, G.-N. Han eds., Springer-Verlag, Berlin Heidelberg, 2001, pp. 139-158. Sém. Lothar. Combin., Art. B42e, 22 pp.

[Ja04] Jackson, J.H., A basic-sine and cosine with symbolic solutions of certain differential equations, Proc. Edinburgh Math. Soc., 22 (1904), pp. 28-39.

[Po89] Poupard, Christiane, Deux propriétés des arbres binaires ordonnés stricts, Europ. J. Combin., 10 (1989), pp. 369-374.

[Pr00] Prodinger, Helmut, Combinatorics of geometrically distributed random variables: new $q$-tangent and $q$-secant numbers, Int. J. Math. Math. Sci., 24 (2000), pp. 825-838.

[Pr08] Prodinger, Helmut, A Continued Fraction Expansion for a $q$-Tangent Function: an Elementary Proof, Sém. Lothar. Combin., B60b (2008), 3 pp.

[Ri58] Riordan, John, An Introduction to Combinatorial Analysis, John Wiley, New York, 1958

[S107] Sloane, N. J. A., On-line Encyclopedia of Integer Sequences, http://www.research.att.com/ njass/sequences/.

[St99] Stanley, Richard P., Enumerative Combinatorics, vol. 2, Cambridge University Press, 1999.

Dominique Foata

Institut Lothaire

1, rue Murner

F-67000 Strasbourg, France

foata@math.u-strasbg.fr
Guo-Niu Han

I.R.M.A. UMR 7501

Université Louis Pasteur et CNRS

7, rue René-Descartes

F-67084 Strasbourg, France

guoniu@math.u-strasbg.fr 